\title{
Oxygen: The Missing Element in Low-Income Countries
}

\author{
Kelly McQueen ${ }^{1}$
}

Published online: 22 October 2015

(C) Société Internationale de Chirurgie 2015

As surgeons and anesthesiologists, primarily from high resource settings or urban areas in low- and middle-income countries (LMICs), we care for patients expecting a baseline of available resources. Surveys and analyses in the past decade revealed critical deficiencies in basic resources for safe surgery and anesthesia in many operative settings in LMICs prompting important safety initiatives and a call to action to insure that processes and equipment are in place for the best surgical care in every setting. Improvements from the World Health Organization Surgical Safety Checklist [1] and the Global Oximetry Initiative [2] have impacted patient safety and saved lives. One essential, life giving and saving element is still missing-oxygen.

Oxygen is ubiquitous in the atmosphere, averaging $21 \%$ of the air we breathe. Its presence sustains life and in the history of medicine increasing the basal percentage of oxygen has improved some health states, improved outcomes, and saved lives. On the Periodic Table, oxygen is element \#16, and harnessing and producing oxygen has been successful since 1903 [3]. While its cost-effectiveness has never been challenged, it is considered basic, essential, and critical to the function of every health care system. In spite of the fundamental role of oxygen to healthcare, it is often overlooked, especially in low-income countries.

Oxygen was overlooked by the World Health Organization (WHO) as an Essential Medicine [4] until 2013. Even so, most physicians and especially anesthesiologists, routinely assume the availability of oxygen, and depend on

Kelly McQueen

kamcqueen@gmail.com

1 Departments of Anesthesiology and the Global Initiative, Vanderbilt University, Nashville, USA oxygen as a treatment and standard of care. Many organizations also make this assumption. Even the Surgical Safety Checklist assumes the availability of oxygen by referring to it only through reference to Anesthesia Guidelines within the checklist. But the reality is that at most first level hospitals in LMICs, many of which are prepared to deliver emergency surgery, access to supplemental oxygen is unpredictable and limited. And as such, anesthesia is routinely delivered on room air, roughly $21 \%$ oxygen [5].

In a new era of Global Surgery, as many governments and hospitals gear up for the provision of emergency and essential surgery for the Billions who have lacked access to surgical care for decades, we must raise awareness and advocate for predictable access to supplemental oxygen when needed. This Call to Action goes out to those successfully advancing patient safety through the Surgical Safety Checklist, which calls for a functional pulse oximeter to be on the patient prior to every surgery. The checklist assumes the availability of supplemental oxygen as part of the anesthesia guidelines. This assumption is aspirational and important, but not realized [6].

In the last decade, those surveying surgical and anesthesia infrastructure have documented the presence and absence of oxygen, and while these surveys are not comprehensive, they estimate that $50 \%$ of the time or less, supplemental oxygen is not available or unpredictably available [7]. Many well-intended physicians, non-governmental organizations, and those planning the scale up to more surgical care may have missed this small, but critical reality while focusing on the bigger picture of providers, equipment, monitors, and, of course, essential medicines. Supplemental oxygen is the missing element in a majority of operative settings in LMICs, and nowhere is it more 
greatly missed then by our surgical patients in need of increased oxygen concentrations for best surgical outcomes, including survival.

Sound the alarm. Alert the media. Call governments, health care systems, donors, and providers to action(Supplemental) Oxygen is inexpensive, life supporting and saving, and essential to safe anesthesia and surgery.

\section{References}

1. The WHO Surgical Safety Checklist. http://www.who.int/patient safety/safesurgery/checklist/en/ Accessed 14 Aug 2015
2. Thoms G, McHugh G, O’Sullivan E (2007) Global oximetry initiative. Anaesthesia 62((Supp)):75-77

3. Anesthesia and Perioperative Care (2015) Essential surgery, vol 1. Disease Control Priorities in Developing Countries, New York

4. World Health Organization Essential Medicines. http://www.who. int/mediacentre/news/releases/2015/new-essential-medicines-list/en Accessed 14 Aug 2015

5. Hodges S, Mijumbi C, Okello M et al (2007) Defining Anaesthesia services in developing countries: defining the problems. Anaesthesia 62((Supp)):4-11

6. McQueen K, Coonan T, Ottaway A et al (2015) The bare minimum: the reality of global Anesthesia and patient safety. World J Surg 39:2153-2160

7. Hadler R, Kushner A, McCunn M (2013) Anesthesia Capacity at health facilities in 17 low and middle-income countries. Alliance for Surgery and Anesthesia Presence (ASAP) abstract, Durham, NC, USA 